\title{
Trans-Interfacial Polymerization and Matter Transport Processes in Epoxy-Alumina Nanocomposites Visualized By Scanning Brillouin Microscopy
}

\author{
R. Sanctuary, ${ }^{* \dagger}$ M. Philipp, ${ }^{\dagger}$ J. Kieffer, ${ }^{\ddagger}, \|$ U. Müller, ${ }^{\dagger}$ W. Possart,${ }^{\S}$ and J. K. Krüger ${ }^{\dagger}$ \\ Laboratoire de Physique des Matériaux, University of Luxembourg, Grand-Duchy of Luxembourg, \\ Department of Materials Science and Engineering, University of Michigan, Ann Arbor, Michigan, and \\ Werkstoffwissenschaften, Universität des Saarlandes, Saarbrücken, Germany
}

Received: March 3, 2010; Revised Manuscript Received: May 10, 2010

\begin{abstract}
The structural developments in the vicinity of the interface between the reactants of an epoxy are investigated using time- and space-resolved scanning Brillouin microscopy. The hypersonic profile across the phase boundary evolves with strong spatial asymmetry and exhibits erratic behavior within the resin-rich region, which is attributed to a complex interplay between matter transport, dissolution, polymerization, and molecular unravelling process. The presence of alumina nanoparticles in the resin changes the character of these matter transport and reaction processes significantly. On the one hand, the nanoparticles act as transport barriers, hindering the mixing of the reactive components; on the other hand they seem to have a catalytic influence on the epoxy polymerization under certain circumstances. Their transport against gravity is tentatively attributed to gradients in surface tension.
\end{abstract}

\section{Introduction}

Epoxies belong to a technically important class of reactive network-forming polymers that are predominantly used as adhesives. ${ }^{1-10}$ Thus, the mechanical properties of this material class are of special importance. These mechanical properties can be significantly improved by adding nanoparticles to the epoxy matrix. ${ }^{2-10}$ This improvement is generally attributed to a three-phase structure where epoxy interphases, which differ from bulk epoxy, are formed around the nanoparticles. ${ }^{1-3}$ However, the existence of interphases in epoxies does not seem to be restricted to nanocomposites. Already for pristine epoxies, heterogeneous structures of macroscopic dimensions ranging between some tens of nanometers and micrometers and differing in their mechanical stiffness have been reported in literature. ${ }^{11-16}$ Note that the appearance of such heterogeneities suggests the existence of interphases between the related epoxy structures. In the context of this paper, an interphase consists of epoxy that connects distinct adjacent domains of homogeneous material. Domains differ based on their physical and chemical constitution; they may consist of the pure epoxy or contain nanoparticles in the polymer. Consequently, the structures within interphases differ from those of bulk epoxy domains and generally exhibit property gradients. Note that domain characteristics and the contrast between domains may appear differently depending on the materials property used to identify them. For example, the existence of optically visible heterogeneities could not be confirmed by hypersonic velocity measurements, ${ }^{17}$ whereas Brillouin microscopy on the same sample revealed acoustic heterogeneities roughly a thousand times smaller than those seen optically. ${ }^{15,17}$

* To whom correspondence should be addressed. Tel: +352 466644 6326. Fax: +352466 644 6331. E-mail: roland.sanctuary@uni.lu.

$\uparrow$ University of Luxembourg.

* University of Michigan.

§ Universität des Saarlandes.

"Sabbatical at the Laboratoire de Physique des Matériaux, University of Luxembourg, Luxembourg.
Taking into account the formation of specific interphases around the nanoparticles in epoxies, the challenging question arises as to what extent the initial sequence of mixing the nanoparticles into one of the epoxy reactants influences the formation of heterogeneities. Refractive index measurements have recently shown that the formation of epoxy networks may strongly depend on the mixing sequence of the three components, ${ }^{8}$ the resin, the hardener, and the nanoparticles. Significant differences between the refractive indices are observed when nanoparticles are dispersed in the resin before being mixed with the hardener, or vice versa, nanoparticles are first added to the hardener before being mixed with the resin. These differences even increase with the ongoing chemical reaction, indicating that for the same overall composition of resin, hardener and nanoparticles significantly different final network structures are formed. The transient and the final structures, including those of the interphases, seem to depend sensitively on the molecular species that are initially in contact with the nanoparticles. That there exist specific interactions between nanoparticles and one or the other of the epoxy educts is well-known. ${ }^{18}$ The real surprise is that, depending on the mixing sequence, these interactions cause the growth of different network structures upon polymerization. This result suggests that the presence of alumina nanoparticles increases the tendency to form metastable thermodynamic states in nanocomposites compared to the pure epoxies.

The origin of the heterogeneities, inhomogeneities and possibly metastable states is far from being understood. This also holds true for the genesis of the polymer network accompanied possibly by gelation and/or vitrification, the formation of the different kinds of interphases, ${ }^{2,8,17}$ and their feedback to the aforementioned phase transitions. This lack of understanding might be due to the complex interplay between physicochemical processes such as chemical reactions of resin, hardener and larger oligomers, their interactions with nanoparticles, and the transport of these objects during the formation of the polymeric network. 
To gain better insight into the subtleties of network formation, we introduced scanning Brillouin microscopy ${ }^{19,20}$ (SBM) as a method capable of probing molecular acoustic changes associated with the chemical reactions and molecular transport at the phase boundary between the hardener and the resin. An initial set of data for the epoxy resin diglycidylether of bisphenol A (DGEBA) and the hardener diethylene triamine (DETA) has recently been published in a letter. ${ }^{20}$

In the current article, we do not only deepen the interpretation of these measurements, but we also compare them with SBM measurements performed near the planar phase boundary between DETA and DGEBA filled with alumina nanoparticles. By carefully layering DETA on top of the two resins and by taking into account the smaller mass density of DETA compared to either resin, we artificially created interfaces at which the reacting species were juxtaposed with fine spatial definition, allowing us to closely examine the rate phenomena associated with polymerization and molecular transport across the initial phase boundary.

Because of the known demixing tendencies of DETA and DGEBA at the initial phase boundary between resin, respectively filled resin, and hardener a transition from an initial immiscibility to an eventual polymerized state will not appear on short time scales. ${ }^{20,21}$ Previous time-resolved SBM investigations of interfacial reactions have indeed revealed that mixing tendencies between DETA and DGEBA are limited and are eventually overcome through the development of convective flow channels ${ }^{20}$ leading to the formation of a spatially inhomogeneous polymer network. In this article, we refine the investigation of segregation tendencies, diffusive and convective matter transport processes, specific chemical/physical bulk and surface interactions, which all influence the structural developments and may contribute to the structural inhomogeneities in the pure epoxy that are imaged by molecular acoustics. ${ }^{22}$ Another topic of this article is to elucidate how the alumina nanoparticles influence the aforementioned processes in the nanoparticles-loaded system. Finally we comment on the relevance of these acoustic investigations for the curing behavior of intentionally well-mixed epoxy systems of the same overall composition.

\section{Experimental Section}

A. Samples and Sample Preparation. Widely investigated epoxy resin and hardener constituents are used in the present study. 2,8,15,17,20,23 The hardener, diethylene triamine (DETA), which possesses a density of $0.96 \mathrm{~g} / \mathrm{cm}^{3}$ at $295 \mathrm{~K}$, was purchased from Fluka. Diglycidylether of bisphenol A, DGEBA DER 331 from DOW Europe, was utilized as the resin. This DGEBA resin contains a small amount of DGEBA dimers and trimers to hinder the room temperature crystallization of the monomers. The melting temperature of this crystalline form of DGEBA is about $315 \mathrm{~K}$. The mass density of the supercooled DGEBA is 1.16 $\mathrm{g} / \mathrm{cm}^{3}$ at $295 \mathrm{~K}$. The alumina nanoparticles Aeroxide Alu C were purchased from Evonik Industries. According to the manufacturer, the primary particles are not surface treated and have an average diameter of $13 \mathrm{~nm}$. van der Waals forces and sintering are responsible for the formation of differently sized aspheric percolated but porous alumina clusters. The linear dimension of some clusters exceeds $300 \mathrm{~nm} .{ }^{3}$ By mechanically dispersing the alumina nanoparticles in the DGEBA resin, the average cluster size was reduced considerably below $100 \mathrm{~nm} .^{3}$ To yield translucent samples to be studied by Brillouin spectroscopy, the alumina concentration of 4.6 mass \% was chosen. Before filling the pure or nanoparticle-loaded resins into glass cuvettes $\left(1 \times 1 \times 4 \mathrm{~cm}^{3}\right)$ to a level of $2 \mathrm{~cm}$, they were degassed to

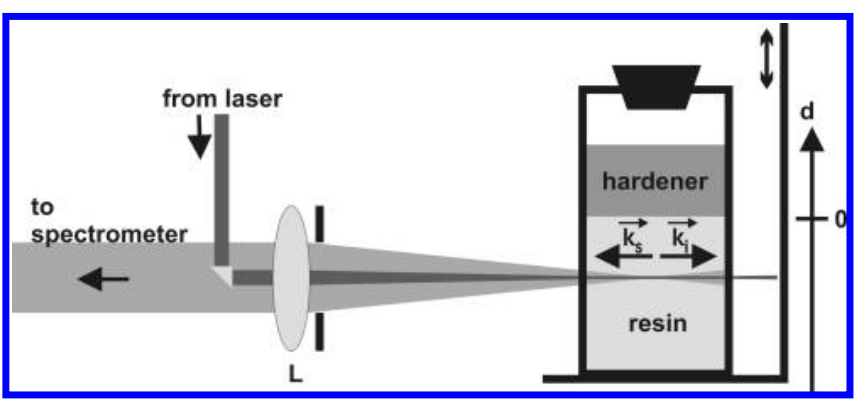

Figure 1. Schematic view of the backscattering geometry and the cuvette on the scanning stage. $\overrightarrow{\mathrm{k}}_{\mathrm{i}}$ and $\overrightarrow{\mathrm{k}}_{\mathrm{s}}$, wave vector of the incident and scattered laser light; L, lens; $d$, vertical axis fixed to the cuvette coordinate system with origin at the initial phase boundary between the hardener and the resin. Cuvette dimensions: $1 \times 1 \times 4 \mathrm{~cm}^{3}$.

remove any air bubbles. Afterward a ca. $1.5 \mathrm{~cm}$ thick layer of DETA was carefully stacked on top of the denser resin before sealing the cuvettes with lids.

In the following, the sample containing pure DGEBA resin is designated as " $\mathrm{R} / \mathrm{H}$ " (resin/hardener) and the one containing the alumina filled resin as "RN/H" (resin, nanoparticles/ hardener).

B. Scanning Brillouin Microscopy. Brillouin spectroscopy is an optical measurement technique that gives access to the hypersonic properties of optically transparent or translucent samples. ${ }^{24,25}$ It is especially well suited for our purpose as it provides spatially and temporally resolved information about the bulk properties of the sample, while being entirely nondestructive.

This technique is based on the inelastic light scattering of laser light by thermal phonons. ${ }^{26,27}$ Applying the law of energy conservation to this scattering process allows one to relate the hypersonic frequency $f$ of the phonons that are probed to the angular frequencies $\omega_{\mathrm{s}}$ and $\omega_{\mathrm{i}}$ of the scattered and incident light, respectively, according to

$$
\hbar \omega_{\mathrm{s}}=\hbar \omega_{\mathrm{i}} \pm 2 \pi \hbar f
$$

Similarly the law of momentum conservation relates the wavevector $\overrightarrow{\mathrm{q}}$ of the phonon to the wavevectors $\overrightarrow{\mathrm{k}}_{\mathrm{s}}$ and $\overrightarrow{\mathrm{k}}_{\mathrm{i}}$ of the scattered and incident light according to

$$
\hbar \overrightarrow{\mathrm{k}}_{\mathrm{s}}=\hbar \overrightarrow{\mathrm{k}}_{\mathrm{i}} \pm 2 \pi \hbar \overrightarrow{\mathrm{q}}
$$

In the backscattering geometry, shown in Figure 1, the incident and scattered wave vectors $\overrightarrow{\mathrm{k}}_{\mathrm{i}}$ and $\overrightarrow{\mathrm{k}}_{\mathrm{s}}$ are antiparallel. In this scattering geometry only the longitudinally polarized phonon doublet can be measured for an elastically isotropic and homogeneous sample. Backscattering from transverse phonons is forbidden due to symmetry. From the Brillouin spectrum the physically relevant hypersonic frequency $f_{\mathrm{L}}^{180}(\overrightarrow{\mathrm{q}})$ and the temporal acoustic attenuation $\Gamma_{\mathrm{L}}^{180}(\overrightarrow{\mathrm{q}})$ of the acoustic phonon can be obtained by deconvoluting the measured Brillouin doublet, taking into account the instrumental broadening along the optical path. The hypersonic frequency and attenuation correspond to the position and the half width at half-maximum of the Brillouin lines, respectively. The statistical error of the hypersonic frequencies typically lies in the one-tenth of percent regime, whereas that of the attenuation is larger by roughly a factor of 10 . 
If the refractive index $n$ of the material in the scattering volume is known, the longitudinal hypersonic velocity $v_{\mathrm{L}}^{180}$ can be determined according to:

$$
v_{\mathrm{L}}^{180}=f_{\mathrm{L}}^{180} \cdot \Lambda^{180}=f_{\mathrm{L}}^{180} \cdot \frac{\lambda_{\text {Laser }}}{2 n}
$$

where $\Lambda^{180}$ is the acoustic wavelength and $\lambda_{\text {Laser }}=532 \mathrm{~nm}$ the vacuum wavelength of the laser (Coherent) used. Since in this experiment the time and space dependent refractive index $n$ of the scattering volume is not accessible, the phonon wavelengths and the longitudinal sound velocities could only be roughly estimated. For all measurements, independent of the presence of nanoparticles or of the degree of network formation, a constant refractive index of $n_{\mathrm{D}}=1.525$ was chosen. This value corresponds roughly to the mean refractive indices of DGEBA (1.571) and DETA (1.482). ${ }^{20}$ In the frame of this approximation the phonon wavelength is $\Lambda^{180}=174 \mathrm{~nm}$. All sound velocities reported below were estimated on the basis of the measured frequencies $f_{\mathrm{L}}^{180}(t, d)$ and this approximated refractive index value. The maximal error of the sound velocity data obtained by error propagation, that is, $\left(\Delta v_{\mathrm{L}}^{180}\right) /\left(v_{\mathrm{L}}^{180}\right) \approx\left(\Delta f_{\mathrm{L}}^{180}\right) /\left(f_{\mathrm{L}}^{180}\right)+(\Delta n) /(n)+$ $\left(\Delta \lambda_{\text {Laser }}\right) /\left(\lambda_{\text {Laser }}\right) \approx(\Delta n) /(n)$, can be as large as $7 \%$.

All measurements were performed with a modified six-pass tandem Brillouin spectrometer of the Sandercock type $e^{24,25,28}$ at $295 \pm 0.5 \mathrm{~K}$. Details of the experimental setup are given in the literature. ${ }^{19,20,28}$ The laser power was kept below $5 \mathrm{~mW}$ to avoid heating of the scattering volume. The needed spatial resolution for the current investigations is obtained by using the SBM setup shown in Figure 1. By using microscope objective lenses to focus the incident laser light and to collect the scattered light and by advancing this focal volume along the length coordinate of the sample, the hypersonic velocities and attenuations could be determined in the material with micrometer spatial resolution. The technical challenge with capturing the structural developments in this epoxy system, which are governed by matter transport and chemical reaction phenomena, was to simultaneously achieve adequate spatial and temporal resolution. As a compromise that provides sufficient spatial coverage, while keeping the time for each scan short compared to the characteristic relaxation time of the overall structural development, the SBM measurements were performed at spatial increments of 20 to $200 \mu \mathrm{m}$ over roughly $d=15 \mathrm{~mm}$. To obtain reliable phonon spectra, the accumulation time was typically $15 \mathrm{~s}$ for the R/H sample and about $30 \mathrm{~s}$ for the RN/H sample. The increased accumulation time for the RN/H sample results from several additional physical mechanisms influencing the genesis of these spectra: the reduced scattering cross section of the alumina loaded DGEBA compared to pure DGEBA, and the optical/acoustic Mie scattering of the laser light/acoustic phonons by the alumina clusters. ${ }^{26,29,30}$ The vertical scanning of the cuvette was achieved by means of an electronically controlled scanning stage from Owis. As shown in Figure 1, the origin of the $d$-axis was chosen at the position of the initial phase boundary between the pure or filled resin and the hardener. Recording spectra in the immediate vicinity of the phase boundary was possible because the meniscus at this interface is almost flat. The scattering volume corresponded to the laser beam's volume within the cuvette and had a length of $10 \mathrm{~mm}$. Because the incident beam was focused near the center of the cuvette, the lateral dimension of the laser beam was not constant but varied between estimated 10 to $50 \mu \mathrm{m}$. So the Brillouin signal contained acoustic information that was spatially averaged over this scattering volume. Acoustic heterogeneities in the scattering volume could be noticed if their dimensions were larger than the acoustic wavelength and the difference of the frequencies involved could be resolved by the spectrometer. In that case, several phonon doublets could be recorded.

\section{Results and Discussion}

A. Results. An essential prerequisite for SBM investigations of trans-interfacial polymerization, matter transport processes, and accompanying structural developments in the vicinity of the initial phase boundary, or initial interface, is the ability to carefully stack DETA either on top of DGEBA, or on DGEBA filled with alumina nanoparticles. To the best of our abilities we avoided mixing of the reactants at the interface during this careful stacking. On the basis of visual inspection, a planar phase boundary was created. The time for sample preparation and proper placement of the cuvette in the SBM setup is a few minutes, shifting the start time of the hypersonic measurements by that period relative to the start of the physicochemical processes that underlie the structural development.

The position of the initial interface is at $d=0 \mathrm{~mm}$. The "time" of each Brillouin scan is assigned to the moment when the position of the scattering volume passes the position of the initial interface. After sample preparation for the $\mathrm{R} / \mathrm{H}$-sample, 24 spatial scans were performed during $43 \mathrm{~h}$, and for the RN/Hsample 42 spatial scans were performed during $95 \mathrm{~h}$. For the sake of clarity, only part of the scans are shown here; these are representative for all measured data sets. For the purpose of identifying the most significant behaviors in the structural evolution of our specimens, it is useful to present data for the short-, intermediate-, and long-time phases of the experiment successively. Short-time refers to the first $3 \mathrm{~h}$, intermediatetime to the period between 10 and $23 \mathrm{~h}$, and long-time to the period longer than $40 \mathrm{~h}$.

Figure 2a shows longitudinal hypersonic velocity/frequency data $v_{\mathrm{L}}^{180} / \mathrm{fL}_{\mathrm{L}}^{80}$ for early spatial scans performed between 0.5 and $3 \mathrm{~h}$ on both samples. According to our recent investigations, the hypersonic velocity of DETA is $1830 \mathrm{~m} / \mathrm{s}$ and that of DGEBA is $2540 \mathrm{~m} / \mathrm{s}$ at ambient temperature. ${ }^{20}$ Because of the rather small concentration of nanoparticles, the sound velocity of the alumina-filled DGEBA, which is $2560 \mathrm{~m} / \mathrm{s}$, exceeds that of pure DGEBA by only $20 \mathrm{~m} / \mathrm{s}$. During the first half hour of material transport and chemical attack, only small changes in the sound velocity profile near the interface are noticeable. As can be seen in Figure 2a, sound velocities differ from the baseline of the respective pristine material only within 1 or 2 $\mathrm{mm}$ of the DETA-rich region (i.e., for $d>0 \mathrm{~mm}$ ). Obviously macroscopic interphases with thicknesses of about $1 \mathrm{~mm}$ and 2 $\mathrm{mm}$ have been formed, which are predominantly located in the DETA-rich region. After three hours, these macroscopic interphases have expanded only to 2 respectively $3 \mathrm{~mm}$ into the DETA-rich compartment.

Toward the latter part of the first three hours, we also indirectly observe by changes of the acoustic properties small amounts of DGEBA molecules and alumina nanoparticles being removed from the alumina-loaded resin surface and dispersed in the DETA-rich compartment. The convective transport of alumina particles against gravity in $\mathrm{RN} / \mathrm{H}$ samples is indeed evident from visual inspection (see Figure 6b). Opposite to the observations for the R/H samples, after stacking DETA on top of the RN-resin a "milky" cloud slowly develops at the phase boundary and propagates upward into the transparent hardener area. The milky cloud is attributed to Mie scattering by aggregates of nanoparticles embedded in DGEBA having diameters above $100 \mathrm{~nm}$. This suggests that nanoparticles are 

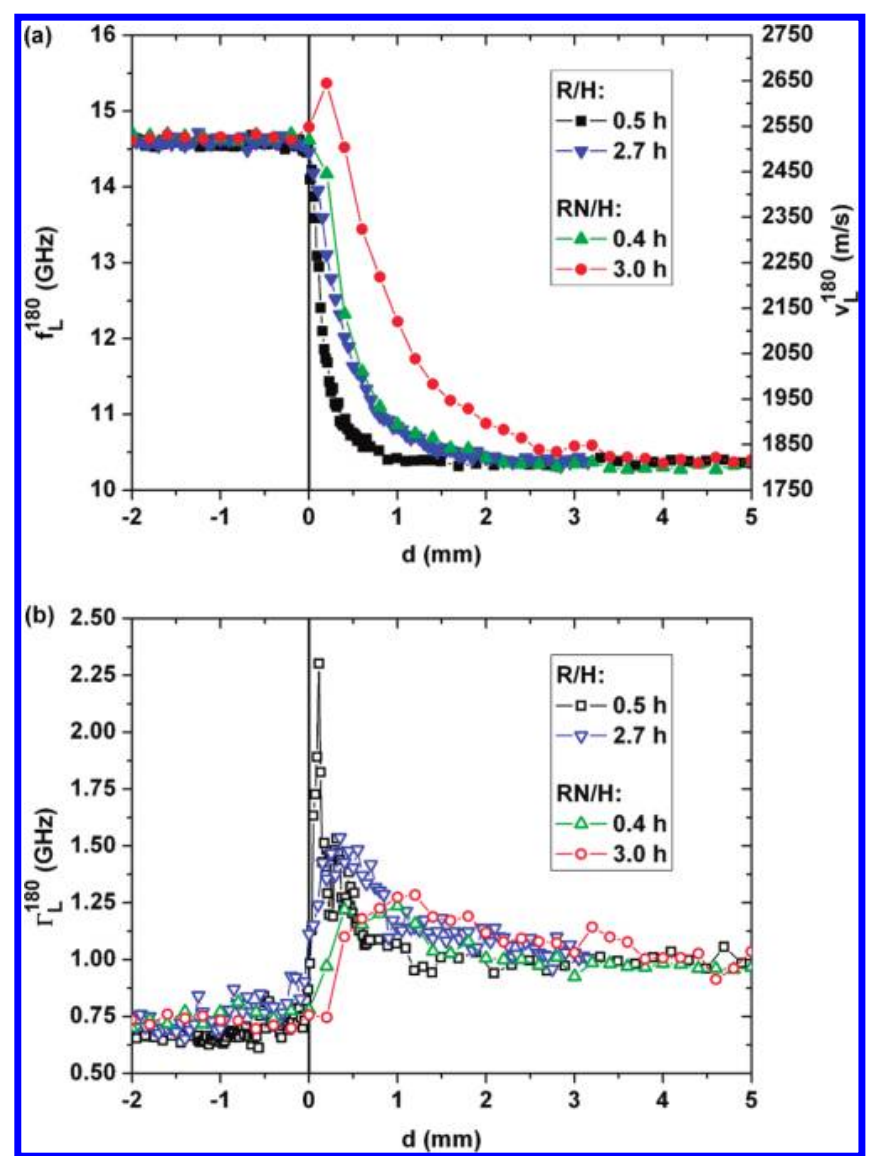

Figure 2. Spatial evolution of (a) the hypersonic frequency $f_{\mathrm{L}}^{180} /$ velocity $v_{\mathrm{L}}^{180}$ and (b) the hypersonic attenuation $\Gamma_{\mathrm{L}}^{180}$ for the pure $\mathrm{R} / \mathrm{H}$ and the alumina filled RN/H sample after ca. 0.5 and $3 \mathrm{~h}$.

propelled along with DGEBA into the hardener-rich region, where the DGEBA molecules chemically react.

The hypersonic behavior observed at intermediate times in the vicinity of the phase boundaries of the $\mathrm{RN} / \mathrm{H}$ and $\mathrm{R} / \mathrm{H}$ samples is more complex than that at short times. The structural evolution at these relatively long times has to be compared with the almost fully polymerized state of a well-mixed sample of the same composition, cured at the same temperature, after six hours. As can be seen in Figure 3a, at $14.7 \mathrm{~h}$ the RN/H sample shows a strong sound velocity excess peak at the initial phase boundary $(d=0 \mathrm{~mm})$ reaching values usually observed for the glassy state of epoxies. The statistical significance of the data point of maximum hypersonic velocity is underlined by the corresponding hypersonic attenuation, which is strongly reduced (see Figure 3b). Because of a gap in the data points, we cannot say to what extent polymerization takes also place in the DGEBA-rich region. If at all, it is restricted to a layer thickness of less than $0.2 \mathrm{~mm}$. At the same time, a significant hypersonic velocity increase is observed for the DETA-rich side, reflecting progress in chemical reaction. The accompanying hypersonic losses remain roughly at values characteristic of pure DETA and show no distinctive features. Consequently, the dynamic viscosity of the material is still dominated by that of the hardener.

The hypersonic properties of the R/H sample at about eleven hours after bringing the constituents in contact with each other exhibit a behavior starkly different from that of the RN/H sample. In the DGEBA-rich region, in the vicinity of $d=-1$ $\mathrm{mm}$, a sound velocity peak largely exceeding that of pure DGEBA is observed. The associated hypersonic attenuation is
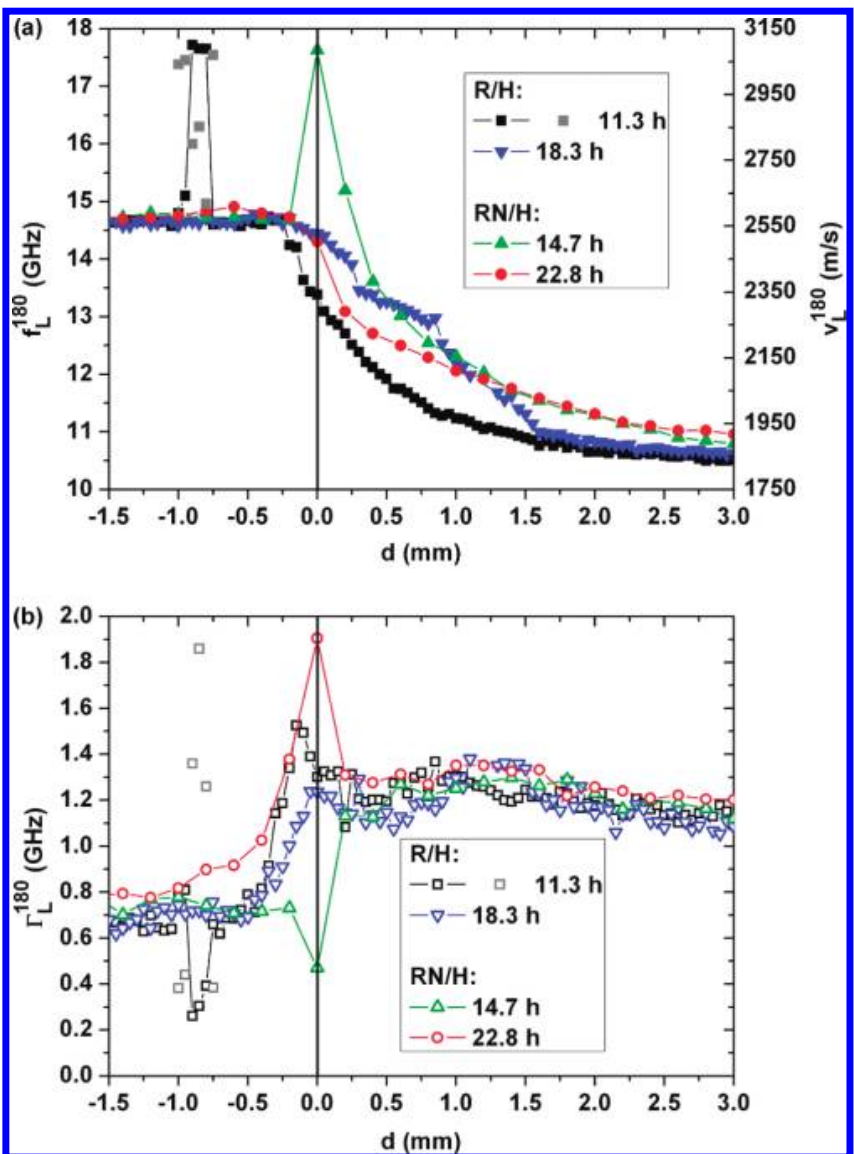

Figure 3. Spatial evolution of (a) the hypersonic frequency $f_{\mathrm{L}}^{800} /$ velocity $v_{\mathrm{L}}^{180}$ and (b) the hypersonic attenuation $\Gamma_{\mathrm{L}}^{180}$ for the pure $\mathrm{R} / \mathrm{H}$ and the alumina filled RN/H sample between 11.3 and $22.8 \mathrm{~h}$.

strongly reduced. A second longitudinally polarized phonon doublet is noticed for six positions of the scattering volume (black and gray squares), which is indicative for the heterogeneity of this zone. ${ }^{20}$ The measures are representative of a chemically induced glassy epoxy embedded in gelatinous epoxy. The real surprise is that immediately above this heterogeneous epoxy network, but definitely within the DGEBA-rich region, hypersonic properties of pure DGEBA are still observed. This result has recently been attributed to convective flow channels of DETA that locally feed DGEBA with the hardener. ${ }^{20}$

After $22.8 \mathrm{~h}$ for the RN/H sample and after $18.3 \mathrm{~h}$ for the $\mathrm{R} / \mathrm{H}$ sample, the hypersonic and consequently the structural profiles have again completely changed along the scanning trace. The excess hypersonic velocity peaks and accompanying hypersonic loss minima at the interface and at $d=-1 \mathrm{~mm}$ for $\mathrm{RN} / \mathrm{H}$ and $\mathrm{R} / \mathrm{H}$ samples, respectively, indicative for glassy epoxy, vanish again. This means that after time periods for which bulk samples with the same overall composition behave stable, in the layered sample even macroscopic glassy epoxy domains are either unstable or are afforded sufficient mobility by the embedding gelatinous network to be displaced from the SBM scattering volume.

In Figure 4, we focus more closely on the time evolution of the excess sound velocity around $d=-1 \mathrm{~mm}$ for the case of pure epoxy. The strong local increase in the sound velocity occurs over a time interval of roughly six hours between 4.6 and $11.3 \mathrm{~h}$, but between 11.3 and $14.0 \mathrm{~h}$ the sound velocities of both the glassy and gelatinous structures strongly diminish in this zone. After $15.5 \mathrm{~h}$, the excess sound velocity around $d=-1 \mathrm{~mm}$ has disappeared; the hypersonic properties are again 

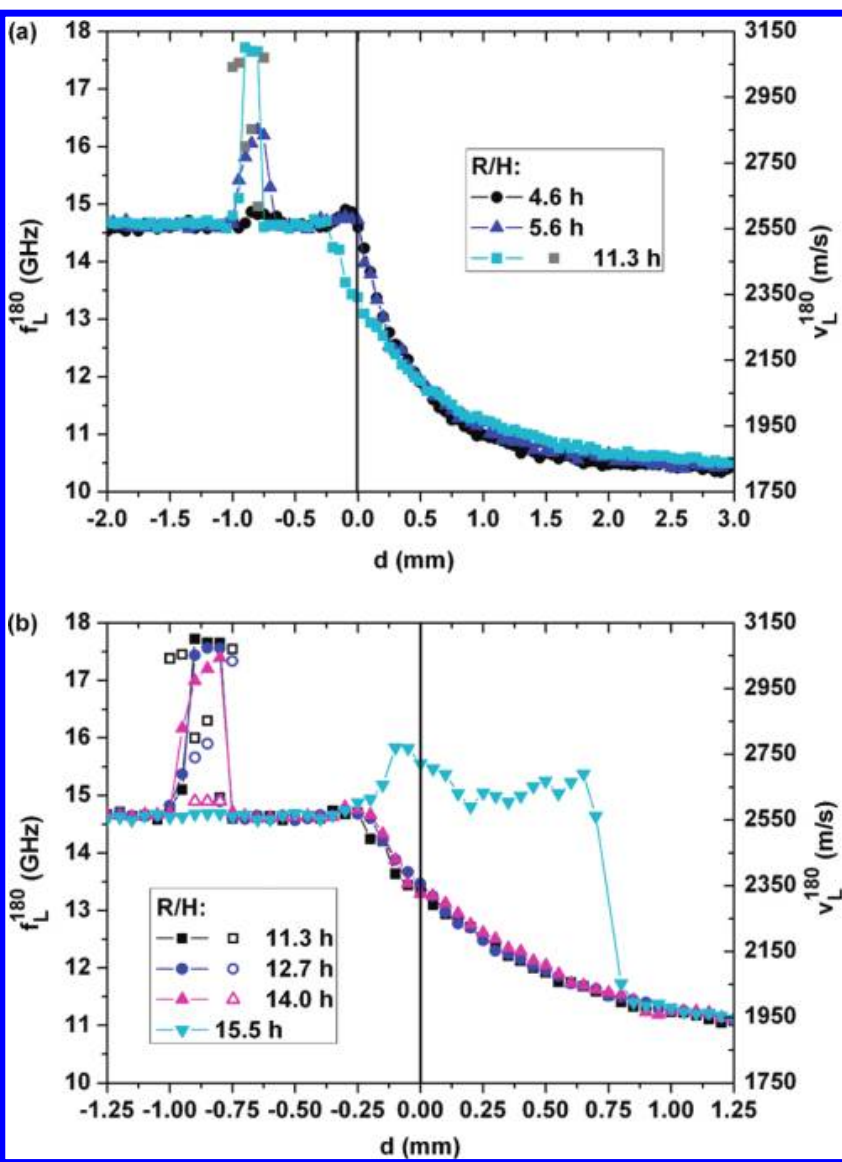

Figure 4. Spatial evolution of the hypersonic frequency/velocity of the R/H sample (a) between 4.6 and $11.3 \mathrm{~h}$, (b) between 11.3 and $15.5 \mathrm{~h}$. Between 11.3 and $14.0 \mathrm{~h}$ two symbols are indicated for a given scan as two phonon doublets are observed for several scattering volumes.

equal to those of DGEBA. Instead, a broad sound velocity shoulder arises in between $-0.25 \mathrm{~mm} \leq d \leq 0.75 \mathrm{~mm}$, indicative for the existence of gelatinous epoxy in this region. As can be seen in Figure 3a, a similar intermittent local peaking of the sound velocity is observed for the RN/H sample. Between 3 and $14.7 \mathrm{~h}$, the hypersonic velocity rose to a significant magnitude at the interface, only to drop down again between 14.7 and $22.8 \mathrm{~h}$.

The evolution of the hypersonic properties and the underlying structural changes in the long-time regime are shown in Figure 5. After $44 \mathrm{~h}$ for the $\mathrm{RN} / \mathrm{H}$ sample the sound properties behave rather inconspicuously along the SBM scanning path. A slight maximum of sound velocity is observed around $d=-0.7 \mathrm{~mm}$ in the resin-rich region. Deeper into the nanoparticle-loaded DGEBA compartment, the measured hypersonic velocities approach their original values again, leaving no reason to assume that the material here is anything but this resin. The maximum sound velocity is much lower than that of a glassy epoxy. An indenter test confirmed that the mechanical consistency of the material at the location of the sound velocity peak area was gelatinous. Above the peak, for $d>-0.7 \mathrm{~mm}$, the sound velocity gradually decreases toward the DETA-rich region. The hypersonic attenuation typical for the latter region has extended slightly into the DGEBA-rich region, including the location where the sound velocity peaks. Note that the nature of the material in the vicinity of the sound velocity peak gradually transitions from gelatinous to liquid in direction toward the DETA-rich region. Seemingly, after more than $40 \mathrm{~h}$ of transinterfacial polymerization material transport is still in progress.
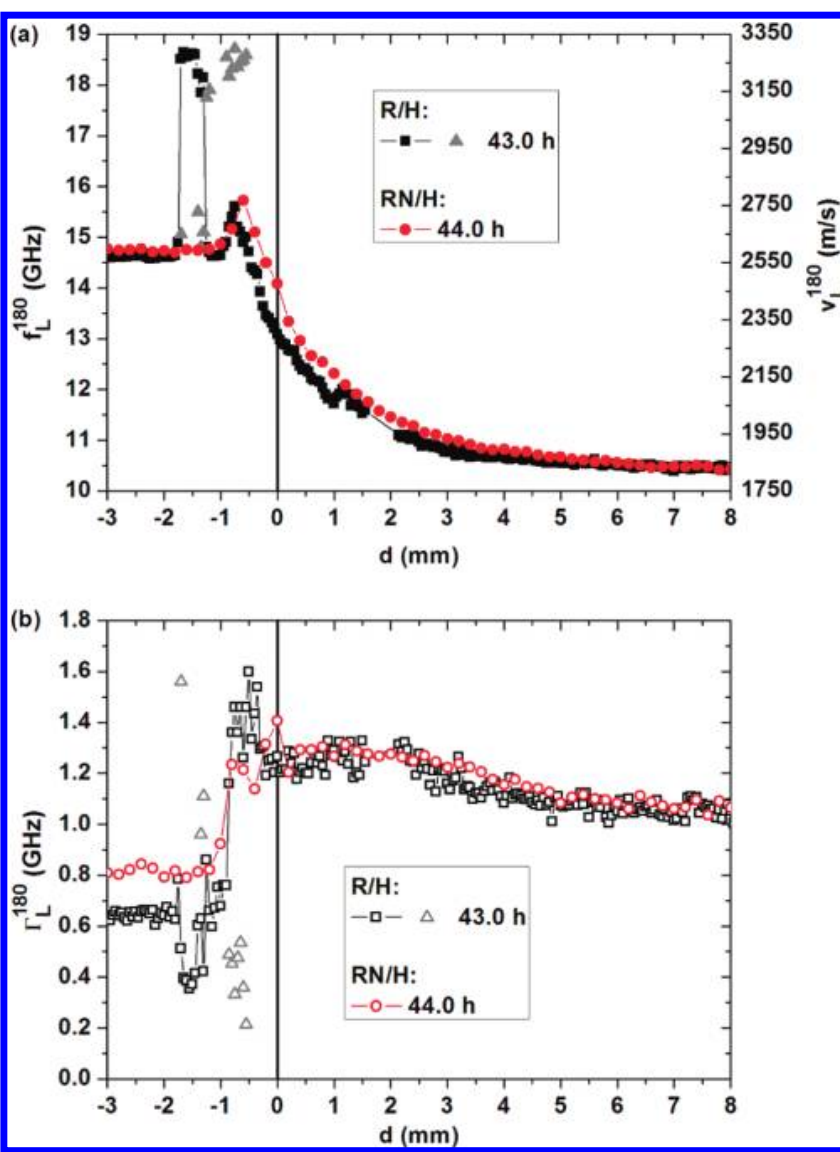

Figure 5. Comparison of the spatial evolution of (a) the hypersonic frequency $f_{\mathrm{L}}^{180} /$ velocity $v_{\mathrm{L}}^{180}$ and (b) the hypersonic attenuation $\Gamma_{\mathrm{L}}^{180}$ for the pure $\mathrm{R} / \mathrm{H}$ and the alumina filled $\mathrm{RN} / \mathrm{H}$ sample after roughly $44.0 \mathrm{~h}$.

As seen in Figure 5, the hypersonic properties of the R/H sample again exhibit after $43 \mathrm{~h}$ several discontinuities along the scanning trace, and even regions in which two longitudinally polarized phonon doublets appear in the Brillouin spectra (squares and triangles in Figure 5). ${ }^{20}$ As before, these acoustic and thus structural heterogeneities can be attributed to the presence of glassy epoxy clusters, partially embedded in a gelatinous matrix on the DGEBA-rich side of the interphase, and in close proximity to the location of the earlier emergence of such clusters. Note that the peak sound velocities are now slightly higher than those observed in this vicinity after about $11 \mathrm{~h}$ of the experiment.

According to Figure 6a, between 45 and $95 \mathrm{~h}$ the interphase in the RN/H-sample is still soft and able to shift by $0.5 \mathrm{~mm}$ to $d=-1 \mathrm{~mm}$. Even after these long times no stable state is reached inside the layered sample. The optical appearance of the RN/H sample is shown in the photograph of Figure $6 \mathrm{~b}$ after $95 \mathrm{~h}$. The milky cloud that was already developing after about $3 \mathrm{~h}$ has in the meantime risen upward, extending $5.5 \mathrm{~mm}$ into the DETA compartment. While the upper border of this cloud is optically rather sharply delineated from the transparent hardener DETA, it is not resolvable in terms of sound properties, which decrease continuously with increasing d. Above $d=0$ $\mathrm{mm}$ the cloud is optically inhomogeneous and less milky than the original alumina loaded DGEBA and the gelatinous network clusters around $d=-1 \mathrm{~mm}$. Especially in the cloud's top part, the nanoparticle concentration is estimated to be considerably below 5 mass \%. As a matter of fact, our acoustic data (see Figure 6a) suggest that the nanoparticles could only emerge from a small volume near the interface between both compartments. 

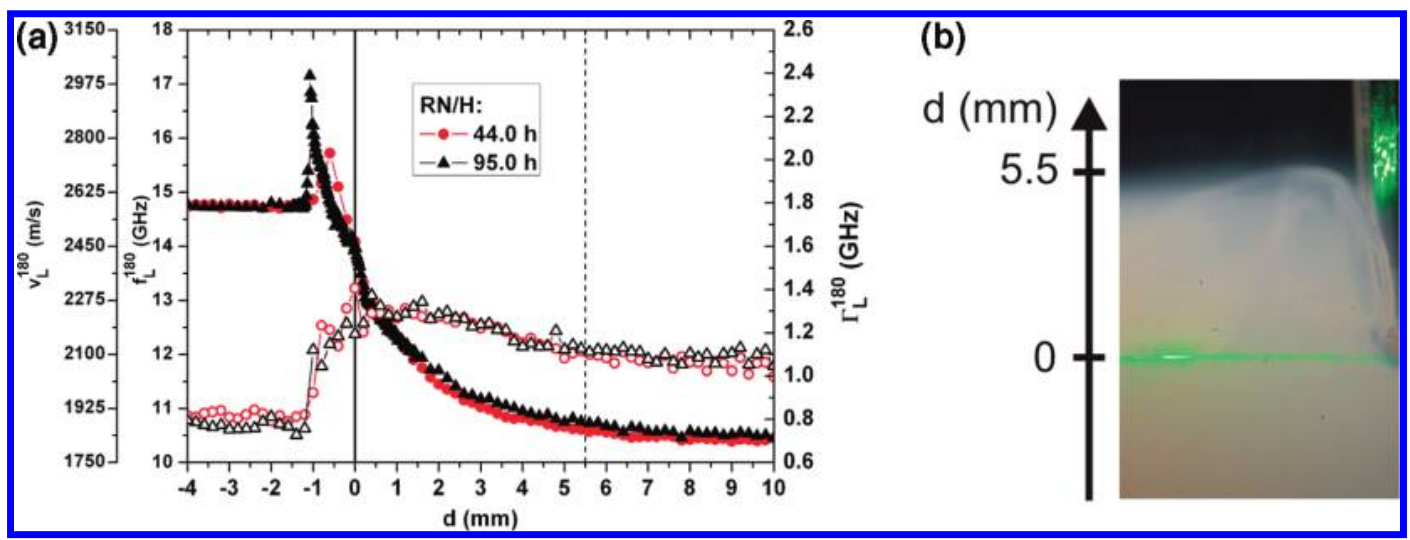

Figure 6. (a) Temporal evolution of the hypersonic frequency $f_{\mathrm{L}}^{180} /$ velocity $v_{\mathrm{L}}^{180}$ and the hypersonic attenuation $\Gamma_{\mathrm{L}}^{180}$ between 44.0 and $95.0 \mathrm{~h}$ for the alumina-filled RN/H sample. Dashed line: position of the white cloud's top shown in Figure 6b. (b) White cloud having propagated from the resin-rich region upward over $5.5 \mathrm{~mm}$ after $95 \mathrm{~h}$.

Furthermore the white cloud is rather translucent in its upper part. On a smaller length scale of about $100 \mu \mathrm{m}$, not resolved on the photograph, the laser beam intensity appears strongly coarse-grained along its trace, indicating the presence of optical inhomogeneities.

B. Discussion. The hypersonic behavior of the layered DGEBA/DETA system is astonishing in several respects. (i) Significant polymerization does not happen immediately at the interface where DETA and DGEBA are mutually exposed to maximum concentrations of their reaction partners; (ii) in the $\mathrm{R} / \mathrm{H}$ system small acoustic heterogeneities, indicative of glassy epoxy network fragments, first appear inside the DGEBA-rich region, away from the interface, and they later disappear again from the scanning trace; and (iii) DGEBA-coated nanoparticles are transported against gravity into the DETA-rich region, faster than pure DGEBA alone or than DETA penetrates into the DGEBA-rich region.

Upon exposing a large concentration of DGEBA to a large concentration of DETA in our layered sample configuration one extreme of the expected behavior might be that the resin and hardener react upon contact and form a glassy layer of epoxy at the original interface, which acts as a diffusion barrier slowing the transport of the large DGEBA molecules and of alumina nanoparticles into the DETA-rich region more than the diffusion of the smaller DETA molecules into the DGEBA-rich region. ${ }^{20}$ Such polymerization would raise the sound velocities on both sides of the interface with respect to those of the original materials. Previous investigations have shown that the hypersonic velocity of a well-cured stoichiometric epoxy glass slightly tops $3000 \mathrm{~m} / \mathrm{s} .{ }^{15,25}$ If, on the other hand, such a diffusion barrier did not build up, mutual diffusion of DETA and DGEBA into one another and subsequent widespread polymerization would be expected. Furthermore, assuming that the alumina nanoparticles in $\mathrm{RN} / \mathrm{H}$ samples did not participate in the transport process, their concentration would increase slightly below the initial phase boundary, which would also affect the sound velocity in that region. However, as revealed in Figure 2a, none of these effects are observed in the DGEBA-rich compartment for either sample type.

Apparently, in the short-time regime the demixing tendency of DGEBA and DETA dominates the general acoustic evolution of the layered sample configuration. The observation that the sound velocity below $d=0 \mathrm{~mm}$ does not change with time, at least during the first three hours, most likely means that DETA does initially not permeate into the DGEBA-rich compartment. The lack of downward DETA transport might be due to the high viscosity of the resin and even more of the filled resin.
The alternative, that is, that the sound velocity decrease for $d<0 \mathrm{~mm}$ due to DGEBA depletion is exactly compensated at all locations by a sound velocity increase due to polymerization, is implausible. Meanwhile, on the DETA-rich side we observe hypersonic changes that are consistent with resin penetrating that compartment. Hence, a small amount of DGEBA is able to surmount the demixing barrier of the reactants at the planar phase boundary and migrate into the DETA compartment. Once DGEBA is molecularly dispersed in between DETA molecules, the chemical environment for the resin resembles that of a wellmixed sample under strongly overstoichiometric conditions, except for a macroscopic concentration gradient. For molecularly dispersed epoxy reactants, the polymerization is not supposed to be hindered. Moreover, measurements of chemical reaction rates show that DGEBA well dispersed in an environment with a strong excess in DETA reacts much faster than DETA dispersed in an excess amount of DGEBA.

According to Figure 2, the short time sound velocity and attenuation behaviors of $\mathrm{R} / \mathrm{H}$ and $\mathrm{RN} / \mathrm{H}$ samples differ only quantitatively from each other. In the DETA rich zone, the excess hypersonic velocities compared to pure DETA are indicative of a structural stiffening and viscosity increase due to polymerization and possibly the formation of network fragments. However, the sound velocity magnitudes are still moderate compared to the $2900 \mathrm{~m} / \mathrm{s}$ measured for a well-mixed sample of the same overall composition after $3 \mathrm{~h}$ polymerization at ambient temperature. ${ }^{20}$ Also, the hypersonic attenuation profiles in the DETA rich region (see Figure $2 b$ ) resemble largely that of pure DETA, indicating that network formation is still incomplete at this point and the viscosity in this region is dominated by DETA molecules. The much smaller hypersonic losses of the RN/H sample compared to the R/H sample close to the interface in the DETA rich phase are probably due to a more pronounced polymerization of the filled sample, which as a consequence behaves frequency clamped. The spatial variation of the hypersonic properties provides a measure of how far DGEBA molecules or nanoparticles have penetrated into the DETA phase. In this context, it should be stressed that measurements of acoustic modes give no direct access to molecular dynamics. Only if molecular dynamics couple to the acoustic strain field (rotational-translational coupling) can this type of dynamics be probed indirectly. ${ }^{26}$

The appearance at intermediate times of glassy epoxy fragments embedded in DGEBA in the $\mathrm{R} / \mathrm{H}$ sample can be attributed to the formation of convective flow channels, allowing for DETA to penetrate into the DGEBA-rich region, where it locally supplies DGEBA with a sufficient amount of hardener 
to achieve the growth of glassy islands. The evolution of convective flow channels and their deep penetration in the viscous DGEBA matrix is a fortiori surprising since the DETArich region adjacent to the initial phase boundary becomes increasingly polymerized thus hindering DETA diffusion toward the DGEBA-rich region. In other R/H samples under the same experimental conditions, the formation of percolated epoxy fragments has also been observed. However their appearance in time and space is unpredictable due to the statistical nature of the driving forces. Such convective channels, however, do not develop deep inside the resin filled with nanoparticles, and hence the formation of glassy epoxy inside the resin compartment of the RN/H system is absent. Figure 4a shows how the sound velocity at the presumed end of the convective flow channel(s) gradually increases with time, demonstrating that indeed the structural feature here evolved via polymerization. Interestingly, DETA that has penetrated DGEBA via such convective microchannels, perhaps akin to viscous fingering, is more reactive than DETA at a large planar interface with DGEBA. Probably at the front edge of these convective channels penetrating DGEBA, a molecular disperse mixture of both reactants is formed, for which polymerization locally leads to an energetically favorable thermodynamic state.

In the R/H sample, the stiff epoxy fragment first appears after about $4.6 \mathrm{~h}$; it exhibits peak sound velocities by $11.3 \mathrm{~h}$ and gradually vanishes over the next $4 \mathrm{~h}$ (see Figure 4). After $43 \mathrm{~h}$ of the experiment, sound velocities that can be attributed to glassy epoxy are again visible in the same vicinity within the scanning trace (see Figure 5a). We can offer two explanations for the disappearance of glassy epoxy fragments from the scanning trace, and possibly a combination of the two applies. One is that after formation, the epoxy aggregate is shifted out of the laser path. Later, the aggregate either shifts again, or more likely, grows back into the laser path. The other possibility would be that the epoxy aggregate is intermittently unravelled. At first sight, both explanations seem implausible. However, additional experiments with glassy epoxies that were generated from a well-mixed sample of DGEBA and DETA, subsequently subjected to a large excess of DETA, have shown that epoxy networks are able to swell and flush out oligomers and larger epoxy fragments visible by eye. Hence, given a continued supply of DETA via convective microchannels, the epoxy aggregates could be purged and unravelled in this fashion. A similar process may take place in the $\mathrm{RN} / \mathrm{H}$ sample, for which the magnitude of the hypersonic velocity significantly decreases close to the initial interface in the DETA-rich region between 14.7 and $22.8 \mathrm{~h}$.

The differences in the structural evolution and molecular acoustic behavior between $\mathrm{R} / \mathrm{H}$ and $\mathrm{RN} / \mathrm{H}$ samples are to be attributed to the presence of the alumina nanoparticles, which cause an increased viscosity that impedes convection deeply into the DGEBA compartment. The obstruction to forming convective transport channels seems to be the principal reason for the absence of glassy structural heterogeneities in the RN/H sample for approximately the first $95 \mathrm{~h}$. As is evident in Figure 2 , the development of the excess sound velocity in the DETArich region is faster for the $\mathrm{RN} / \mathrm{H}$ system than for the $\mathrm{R} / \mathrm{H}$ system. In fact, the hypersonic velocity profile of the RN/H sample measured after $0.4 \mathrm{~h}$ coincides with that measured for the $\mathrm{R} / \mathrm{H}$ sample $2.3 \mathrm{~h}$ later (see Figure $2 \mathrm{a}$ ). This must be associated with the fact that alumina nanoparticle clusters carrying DGEBA molecules are propelled against gravity over macroscopic distances (more than $5 \mathrm{~mm}$ after $90 \mathrm{~h}$ ) into the
DETA-rich compartment. The question then arises as to the driving forces behind this unusual matter transport process.

The possibility that thermal gradients induced by laser heating could be the origin of convective currents is one of the first thoughts that come to mind. However, any measurable heating of the sample by the laser light was avoided by keeping the power at the sample below $5 \mathrm{~mW}$. Evidence that no significant local heating took place is provided by the laser trace shown in figure $6 \mathrm{~b}$, which shows no sign of thermal defocusing. Moreover, the upward transport of alumina nanoparticles that causes the formation of the milky cloud in the DETA-rich region of the RN/H samples also takes place without illuminating the sample by the laser. We successfully repeated this experiment twice. According to an optical inspection, the form of the cloud developed differently for both samples and it seems that the visible onset of the cloud's formation is also irregular in time. If heating through an external source such as the laser can be ruled out, another cause for convective flow could be the exothermal heat production due to the chemical reactions within the sample. However, the whole polymerization process is extremely slow compared to thermal conduction. Thus, only minute temperature fluctuations can be expected, and these would rapidly dissipate. Temperature variations of even a few Kelvin across the scattering volume would also cause thermal defocusing, which is not observed. Hence, given the evidence at hand, we do not believe that thermal gradients are responsible for the upward transport of the alumina nanoparticles.

As a result of DGEBA diffusion into the DETA compartment, gradients of chemical composition, structural character, and physical properties develop. This likely includes gradients of chemical affinity, and consequently of surface tension between the nanoparticles and the DETA-rich liquid they are suspended in. A possible explanation for the upward drift of the alumina nanoparticles is therefore as follows. Initially, these particles are completely wetted by DGEBA and embedded in the bottom compartment of the sample. As DGEBA diffuses into DETA, the top layers of the resin matrix get carried away, slowly exposing the nanoparticles. If the surface tension between DETA and alumina is lower than that between DGEBA and alumina, then, as soon as the nanoparticle is exposed and wetted by a DETA-rich solution, an upward impulse is created that extricates the filler particle from its matrix. Moreover, within the chemical gradient created by the preceding diffusion of DGEBA into DETA, the top of the alumina particle will always be exposed to a solution that is richer in DETA than the bottom. The associated differential between the surface tension at the top and bottom results in a sustained force that propels the nanoparticle upward, against gravity, for as long as the particle is positioned within a chemical gradient. A rough estimate of the pertinent materials properties indicates that surface tension gradients in excess of $1 \mathrm{~kg} / \mathrm{ms}^{2}$ can easily develop when DGEBA diffuses into DETA, which can sustain a terminal velocity of about $5 \times 10^{-7} \mathrm{~m} / \mathrm{s}$ for a particle measuring $1 \mu \mathrm{m}$ in diameter (future publication). A schematic of the underlying mechanism is given in Figure 7. This effect is analogous to that reported by Chaudhury and Whitesides, ${ }^{31}$ where a droplet of water is propelled uphill when placed on an inclined surface that is coated so as to exhibit a gradient of hydrophobicity.

We assume that the alumina nanoparticles are sufficiently close to the DGEBA/DETA interface, so that within the first half hour of the experiment a detectable amount of nanoparticles is exposed at their top hemisphere to DETA molecules. According to Figure 2, the initial network formation in the RN/H sample is much faster than in the $\mathrm{R} / \mathrm{H}$ sample. On the one hand, 


\section{(a)}

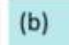

(c)

(d)
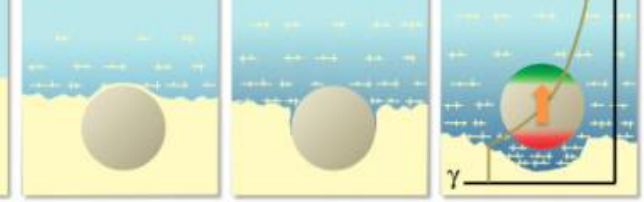

Figure 7. Illustration of the mechanism that causes the extrication of alumina nanoparticles (sphere) from the DGEBA resin (yellow) and the propulsion into the DETA hardener phase (blue). (a) Initial condition of layered filled resin and hardener; (b) DGEBA diffuses into DETA, which erodes the interface and exposes nanoparticles, while a concentration gradient builds up in the DETA phase; (c) DETA wets the upper hemisphere of the particle creating an impulse that dislodges it; (d) the particle is propelled upward due to a surface tension differential caused by the concentration gradient in DETA. Superimposed is a schematic of the surface tension $\gamma$ vs vertical position $z$.

this may be due to the transport of additional DGEBA molecules into the DETA compartment induced by the extrication of the nanoparticles, possibly in form of patches of coating still attached to the side or bottom of the particles. On the other hand, the presence of nanoparticles could catalyze the polymerization. Little is known about the reactivity of DETA and DGEBA in the proximity of the alumina particles but probably, these particles accelerate the chemical reaction of oxirane and amine groups. ${ }^{2,8,18}$ Evidently, the surface tension driven matter transport will only work for as long as the nanoparticles are exposed to sufficiently steep concentration gradients capable of providing the surface tension differential that exceeds the gravitational forces and the increasing friction forces caused by the formation of larger epoxy fragments. Furthermore, the transport of nanoparticles can only occur for as long as the reacting epoxy does not form a percolating network, obstructing the motion of the particles. Eventually, the transport of nanoparticles is blocked by the advancing polymerization in the DETA-rich compartment. Matter transport and polymerization processes beyond $43 \mathrm{~h}$ are probably dominated by the diffusion of DETA and other small molecules through epoxy network structures. Figure 6a proves that transport of the small molecules continues on much longer time scales, optical investigations suggest that no global equilibrium is achieved even over weeks.

The upward transport of alumina nanoparticles is expected to lead to an increasing interface roughness between the resinand hardener-rich compartments. This interface roughness could improve the probability for convective transport channels to form into the RN compartment. However percolated epoxy clusters are not observed in the bottom compartment of the $\mathrm{RN} / \mathrm{H}$ sample within the frame of the given spatiotemporal resolution. Hence, either the high static viscosity of the aluminaloaded DGEBA impedes the formation of convection channels or the convection channels are too short-ranged or short-lived to be detectable.

The above results evoke the question as to what extent they have relevance for the praxis of producing epoxy materials by preparing "well-mixed" batches of resin and hardener through mechanical stirring. On the basis of our findings, rapid polymerization could require molecularly dispersed components, so as to overcome the tendency toward phase separation of the two substances. This occurred most effectively within microchannels, whose size is estimated to be of the order of tens of micrometers. Blending that leaves larger pockets of unmixed components will likely lead to a spatial variation of reaction rates, the formation of convection barriers and an overall inhomogeneous polymerization process across the material.

\section{Conclusions}

Scanning Brillouin microscopy analysis of specimens in which DETA is layered on top of either pure or alumina-filled DGEBA reveals the development of complex morphological heterogeneities during trans-interfacial epoxy polymerization. The observed structural evolutions, as reflected by the materials' acoustic responses, are the result of a complex interplay between chemical reactions and three different kinds of matter transport processes across the phase boundary. (i) DGEBA diffuses into the DETA-rich region much more rapidly than DETA penetrates the DGEBA-rich region, resulting in the formation of network fragments in the DETA-rich region, which causes the stiffness of the liquid to increase to an extent that tapers off with increasing distance from the interface. (ii) Alumina particles embedded in DGEBA, once exposed to DETA, dislodge from the resin and ascend against gravity into the DETA-rich region. We conjecture that the driving force for this particle transport is the vertical surface tension gradient that arises in the liquid when DGEBA diffuses into DETA. Overall, for samples loaded with alumina nanoparticles network formation advances more rapidly into the DETA-rich region than for samples containing only pure resin and hardener. This may be because the upward drifting nanoparticles drag with them additional DGEBA molecules, and/or because the presence of nanoparticles catalytically accelerates the epoxy polymerization. (iii) For the sample containing no nanoparticles, glassy epoxy structures of limited expanse are detected in the scanning trace based on their large sound velocities and their small sound attenuations. These local glassy structures occur at some distance below the initial phase boundary and are generated at these locations as a result of narrow convective DETA streams akin to viscous fingering, at the front end of which the constitutions of DETA and DGEBA are altered so as to overcome phase separation tendencies and polymerize into glassy epoxy. These glassy epoxy aggregates eventually disappear from the scanning trace, most likely due to a convection-induced shift away from the laser path and unravelling of the aggregates. In the nanoparticle-loaded epoxy sample, the formation of convective transport channels is prevented because of the high viscosity and the development of convection barriers in the DGEBA-rich region. For this sample, the scanning Brillouin microscopy investigation was extended to $95 \mathrm{~h}$, revealing extremely slow transport of the different reactive molecular units through the viscous liquid and the gelatinous parts.

Acknowledgment. This work was financially supported by the University of Luxembourg, especially the project "Static and dynamic properties of nanocomposites" and by the National Research Fund of Luxembourg.

\section{References and Notes}

(1) Friedrich, K.; Fakirov, S.; Zhang, Z. Polymer composites. From nano- to macro-scale; Springer: New York, 2005.

(2) Sanctuary, R.; Baller, J.; Zielinski, B.; Becker, N.; Krüger, J. K.; Philipp, M.; Müller, U.; Ziehmer, M. J. Phys.: Condens. Matter 2009, 21, 35118.

(3) Wetzel, B.; Rosso, P.; Haupert, F.; Friedrich, K. Eng. Fract. Mech. 2006, 73, 2375.

(4) Dudkin, B. N.; Zainullin, G. G.; Krivoshapkin, P. V.; Krivoshapkina, E. F.; Ryazanov, M. A. Glass Phvs. Chem. 2008, 34, 187. 131.

(5) Gorbatkina, Y. A.; G, I.-M. V.; M, U. y. T. Polym. Sci. 2007, 49,

(6) Ji, Q. L.; Zhang, M. Q.; Rong, M. Z.; Wetzel, B.; Friedrich, K. J. Mater.Sci. 2004, 39, 6487.

(7) Zhai, L. L.; Ling, G. P.; Wang, Y. W. Int. J. Adhes. Adhes. 2007, $28,23$. 
(8) Philipp, M.; Gervais, P.-C.; Sanctuary, R.; Müller, U.; Baller, J.; Wetzel, B.; Krüger, J. Exp. Polym. Lett. 2008, 2, 546. 2002

(9) Pascault, J. P. Thermosetting polymers; Marcel Dekker: New York,

(10) Petrie, E. M. Epoxy adhesive formulations; McGraw-Hill: New York, 2006.

(11) Giraud, M.; Nguyen, T.; Gu, X.; Van Landingham, M. R. In Effects of stoichiometry and epoxy molecular mass on wettability and interfacial microstructures of amine-cured epoxies; 24th Annual meeting of adhesion society, Williamsburg, VA, February 25-28, 2001; Proceedings, p. 260

(12) Dušek, K. Angew. Makromol. Chem. 1996, 240, 1.

(13) Mijovic, J.; Tsay, L. Polymer 1981, 22, 902.

(14) Takahama, T.; Geil, P. G. Makromol. Chem., Rapid Commun. 1982, 3,389 .

(15) Wehlack, C.; Possart, W.; Krüger, J. K.; Müller, U. Soft Materials 2007, $5,87$.

(16) Wetzel, B. Mechanische Eigenschaften von Nanokompositen aus Epoxydharz und keramischen Nanopartikeln. Ph.D. Thesis, Technische Universität Kaiserslautern, 2005.

(17) Müller, U.; Bactavatchalou, R.; Baller, J.; Philipp, M.; Sanctuary, R.; Zielinski, B.; Alnot, P.; Possart, W.; Krüger, J. K. New J. Phys. 2008, 10, 23031.

(18) Mercier, D.; Rouchaud, M.-G.; Barthés-Labrousse, M.-G. Appl. Surf. Sci. 2008, 254, 6495.

(19) Philipp, M.; Collette, F.; Veith, M.; Seck, P.; Sanctuary, R.; Müller, U.; J, K.; Krüger, J. K. J. Phvs. Chem. B 2009, 113, 12655.

(20) Sanctuary, R.; Philipp, M.; Müller, U.; Possart, W.; Jiménez Riobóo, R. J.; Krüger, J. K. Chem. Phvs. Lett. 2009, 476, 11.
(21) Ellis, B. Chemistry and technology of epoxy resins; Chapman \& Hall: London, 1993.

(22) Schaaffs, W. Molecular acoustics; Springer: Berlin, 1967; Vol. 5.

(23) Possart, W.; Krüger, J. K.; Wehlack, C.; Müller, U.; Petersen, C.; Bactavatchalou, R.; Meiser, A. C. R. Chim. 2006, 9, 60.

(24) Krüger, J. K. Brillouin spectroscopy and its application to polymers. In Optical Techniques to Characterize Polymer Systems; Bässler, H., Ed.; Elsevier: Amsterdam, 1989.

(25) Krüger, J. K.; Alnot, P.; Baller, J.; Bactavatchalou, R.; Dorosz, S.; Henkel, M.; Kolle, M.; Krüger, S. P.; Müller, U.; Philipp, M.; Possart, W.; Sanctuary, R.; Vergnat, C. About the nature of the structural glass transition: An experimental approach. In Ageing and the Glass Transition; Henkel, M., Pleimling, M., Sanctuary, R., Eds.; Springer: Berlin, 2007.

(26) Berne, J. B.; Pecora, R. Dynamic light scattering with applications to chemistry, biology, and physics; Wiley: New York, 1976.

(27) Nye, J. F. Physical Properties of Crystals; Oxford Press: Oxford, 1972.

(28) Krüger, J. K.; Müller, U.; Bactavatchalou, R.; Liebschner, D.; Sander, M.; Possart, W.; Wehlack, C.; Baller, J.; Rouxel, D. Mechanical interphases in epoxies as seen by non-destructive high-performance Brillouin microscopy. In Adhesion - Current research and applications; Possart, W., Ed.; Wiley-VCH: Weinheim, 2005; p 125.

(29) Born, M.; Wolf, E. Principles of Optics; Cambridge University Press: Cambridge, 1999.

(30) Morse, P. M.; Uno Ingard, K. Theoretical Acoustics; Princeton University Press: Princeton, 1968.

(31) Chaudhury, M. K.; Whitesides, G. M. Science 1992, 256, 1539. JP101932H 